LA-UR- $04-7867$

Approved for public release; distribution is unlimited.
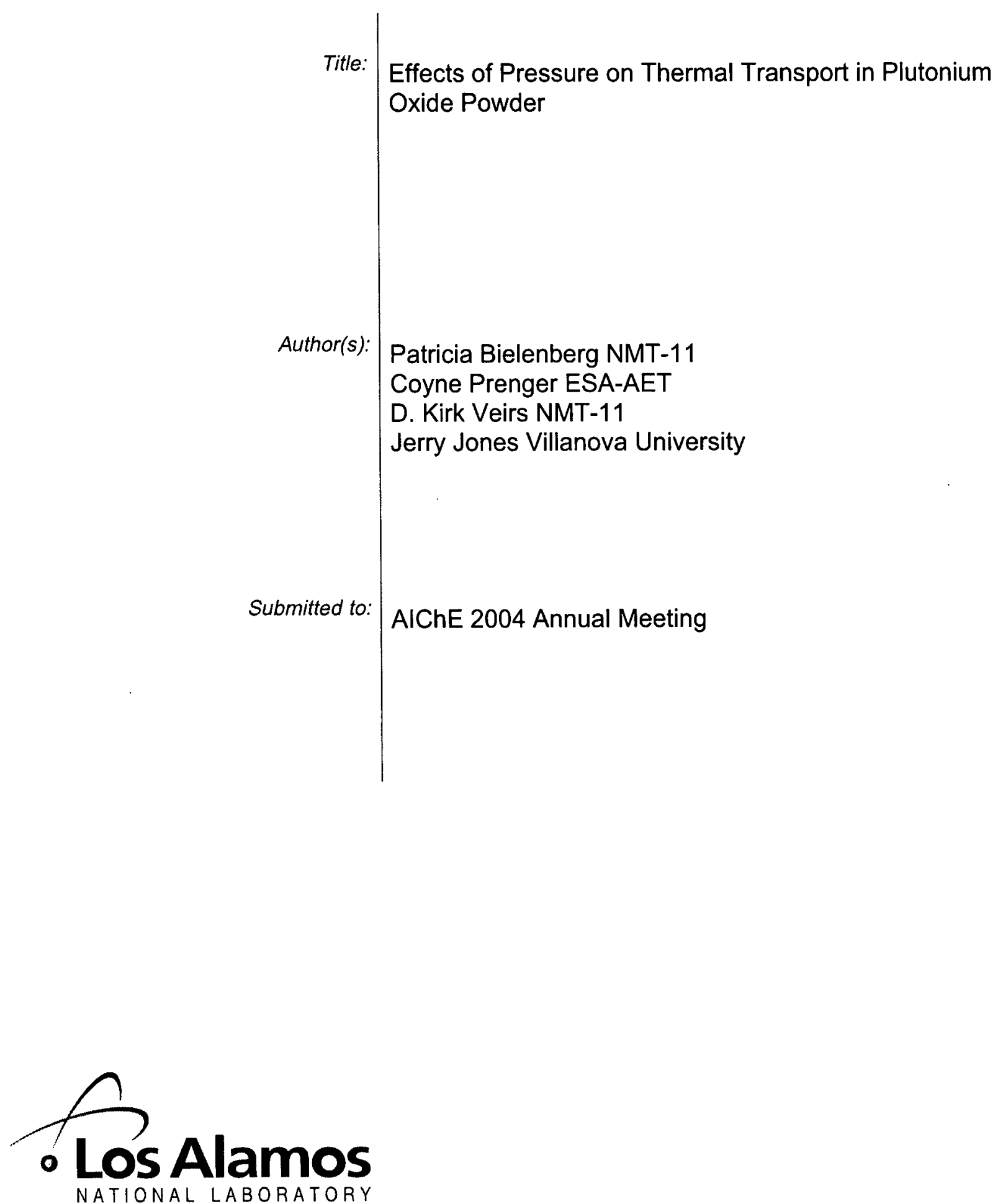

Los Alamos National Laboratory, an affirmative action/equal opportunity employer, is operated by the University of California for the U.S. Department of Energy under contract W-7405-ENG-36. By acceptance of this article, the publisher recognizes that the U.S. Government retains a nonexclusive, royalty-free license to publish or reproduce the published form of this contribution, or to allow others to do so, for U.S. Government purposes. Los Alamos National Laboratory requests that the publisher identify this article as work performed under the auspices of the U.S. Department of Energy. Los Alamos National Laboratory strongly supports academic freedom and a researcher's right to publish; as an institution, however, the Laboratory does not endorse the viewpoint of a publication or guarantee its technical correctness. 
Radial temperature profiles in plutonium oxide $\left(\mathrm{PuO}_{2}\right)$ powder were measured in a cylindrical vessel over a pressure range of 0.055 to $334.4 \mathrm{kPa}$ with two different fill gases, helium and argon. The fine $\mathrm{PuO}_{2}$ powder provides a very uniform self-heating medium amenable to relatively simple mathematical descriptions. At low pressures $(<0.1 \mathrm{kPa})$, the effective thermal conductivity of the powder bed was approximately the same with either helium or argon since the dominant mechanisms are thermal radiation between particles and solid-solid conduction pathways. At high pressures, the effective thermal conductivity of the powder bed is typically assumed in the literature to be dominated by the gas thermal conductivities. However, from experimental measurements at high pressures, the effective thermal conductivity of the powder bed with argon as a fill gas is approximately three times higher than would be predicted from the gas thermal conductivities. Additionally, a significant pressure dependence was measured at pressures greater than atmospheric where the gas thermal conductivity would typically be assumed to be in the continuum limit and independent of pressure.

An analytical model was developed for heat conduction in the fine ceramic powder with conduction pathways in parallel and in series through the gaseous and solid components. Many analytical models in the literature were unsuitable for this system because they make limiting assumptions about the particle dimensions and shape and are developed for packed beds with higher packing fractions. $\mathrm{PuO}_{2}$ powder has small particle sizes (on the order of 1 to $10 \mu \mathrm{m}$ ), random particle shapes, and high porosity so a more general model was required for this system. The model correctly predicts the temperature profiles of the powder over the wide pressure range for both argon and helium as fill gases. The effective thermal conductivity of the powder bed exhibits a pressure dependence at higher pressures because the pore sizes in the interparticle contact area are relatively small (less than $1 \mu \mathrm{m}$ ) and the Knudsen number remains above the continuum limit at these conditions for both fill gases. Also, the effective thermal conductivity with argon as a fill gas is higher than expected at higher pressures because the solid pathways account for over $80 \%$ of the effective powder conductivity. The results obtained from this model help to bring insight to the thermal conductivity of very fine ceramic powders with different fill gases. 


\section{Effects of Pressure on Thermal Transport in Plutonium Oxide Powder}

Patricia Bielenberga, Coyne Prenger ${ }^{b}$, Kirk Veirs ${ }^{a}$, and Jerry Jones ${ }^{c}$

a Los Alamos National Laboratory, Nuclear Materials Technology Division

${ }^{\mathrm{b}}$ Los Alamos National Laboratory, Engineering Sciences and Applications Division

c Villanova University, Dept. of Mechanical Engineering

AIChE Annual Meeting

Austin, TX

November 8, 2004 


\section{Background and Motivation}

- The DOE is storing plutonium dioxide $\left(\mathrm{PuO}_{2}\right)$ powder in storage containers while awaiting final disposition

- The governing storage standard specifies limits on the safe storage of $\mathrm{PuO}_{2}$ containers

- Power generation < $19 \mathrm{~W}$

- Mass of $\mathrm{PuO}_{2}<5.0 \mathrm{~kg}$

- No specific temperature specifications

- Thermal properties of $\mathrm{PuO}_{2}$ in the storage configuration are important because as temperature increases:

- outgassing in the container increases

- fill gas pressure increases

- vapor pressure of water increases

- distribution of water between materials changes

- kinetics of reactions affecting hydrogen gas generation and corrosion changes

9975 container

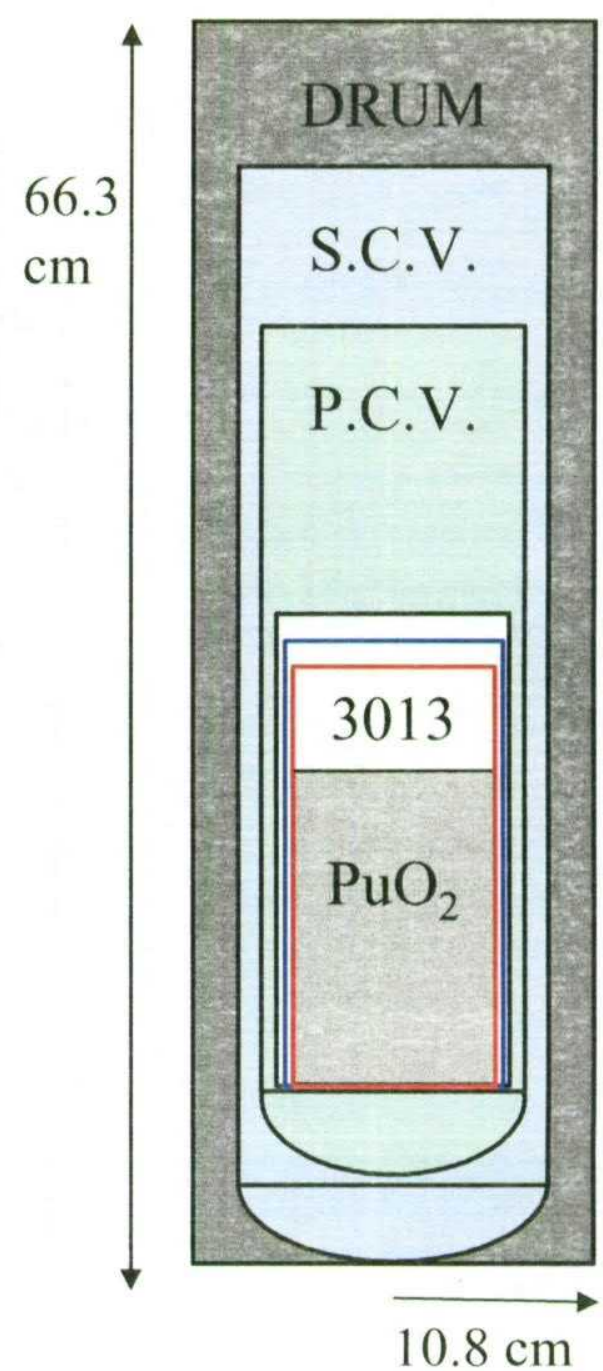




\section{Background and Motivation: Previous modeling efforts}

- Finite element models were developed to predict temperature profiles throughout storage containers ${ }^{1-3}$

- No experimental data for the effective thermal conductivity of the $\mathrm{PuO}_{2}$ powder were available

- Deissler-Eian correlation used to predict effective thermal conductivities

- estimated from solid thermal conductivity $\left(k_{s}\right)$, gas thermal conductivity $\left(k_{g}\right)$, and powder porosity $(\varepsilon)$

- For $k_{\text {eff }}=0.079 \mathrm{~W} / \mathrm{m}-\mathrm{K}$ and $19 \mathrm{~W}$ of $\mathrm{PuO}_{2}$,

- $T_{\max }$ was $229^{\circ} \mathrm{C}$

- radial $\Delta \mathrm{T}$ across the $\mathrm{PuO}_{2}$ bed was $100^{\circ} \mathrm{C}$

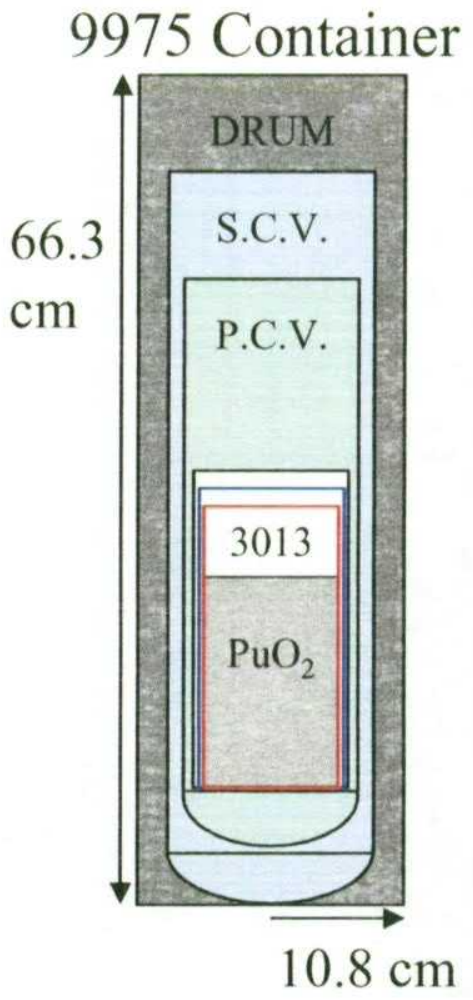

\begin{tabular}{llll}
\hline $\mathrm{PuO}_{2}$ powder thermal conductivity $(\mathrm{W} / \mathrm{m}-\mathrm{K})$ & $T=50{ }^{\circ} \mathrm{C}$ \\
\hline fill gas & $\varepsilon=0$ & $\varepsilon=0.76$ & $\varepsilon=1.0$ \\
\hline Helium & 1.88 & 0.31 & 0.16 \\
Air & 1.88 & 0.082 & 0.027 \\
Argon & 1.88 & 0.061 & 0.019 \\
\hline
\end{tabular}

1. T. D. Knight and R. G. Steinke, Thermal analysis of plutonium materials in British Nuclear Fuels, Ltd., containers, Los Alamos National Laboratory Report, LA-UR-97-1866, 1997. 


\section{Current heat transfer study}

Goal: Provide a better prediction of heat transfer rates in $\mathrm{PuO}_{2}$ powder for use in computing maximum $T, P$ in storage containers

- Experimentally measure temperature profiles in $\mathrm{PuO}_{2}$ powder

- Develop a model for $k_{\text {eff }}$ of the $\mathrm{PuO}_{2}$ powder and compare predictions to experimental data 


\section{$\mathrm{PuO}_{2}$ powder thermal profile measurements}

Radial temperature profiles measured in porous $\mathrm{PuO}_{2}$ at different pressures

- Porosity $=0.774$

- $m_{\text {bed }}=5.00 \mathrm{~kg}$

- Wattage $=10.3 \mathrm{~W}$

- $V_{\text {bed }}=1920 \mathrm{~cm}^{3}$

- Gases: Argon and Helium

- $P$ range $=0.055$ to $334 \mathrm{kPa}$
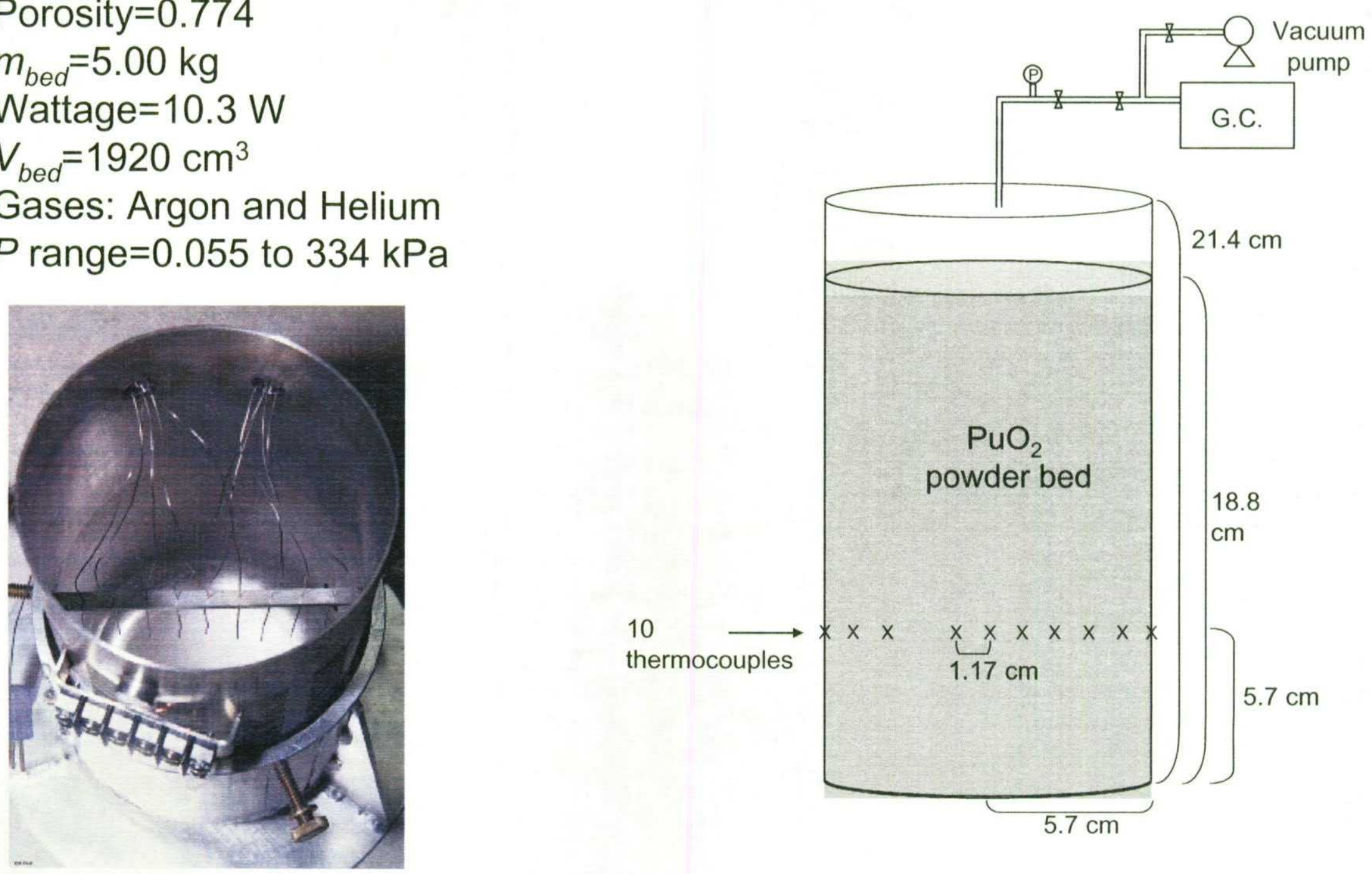


\section{Experimental results for Ar powder}

Radial profiles - Argon powder

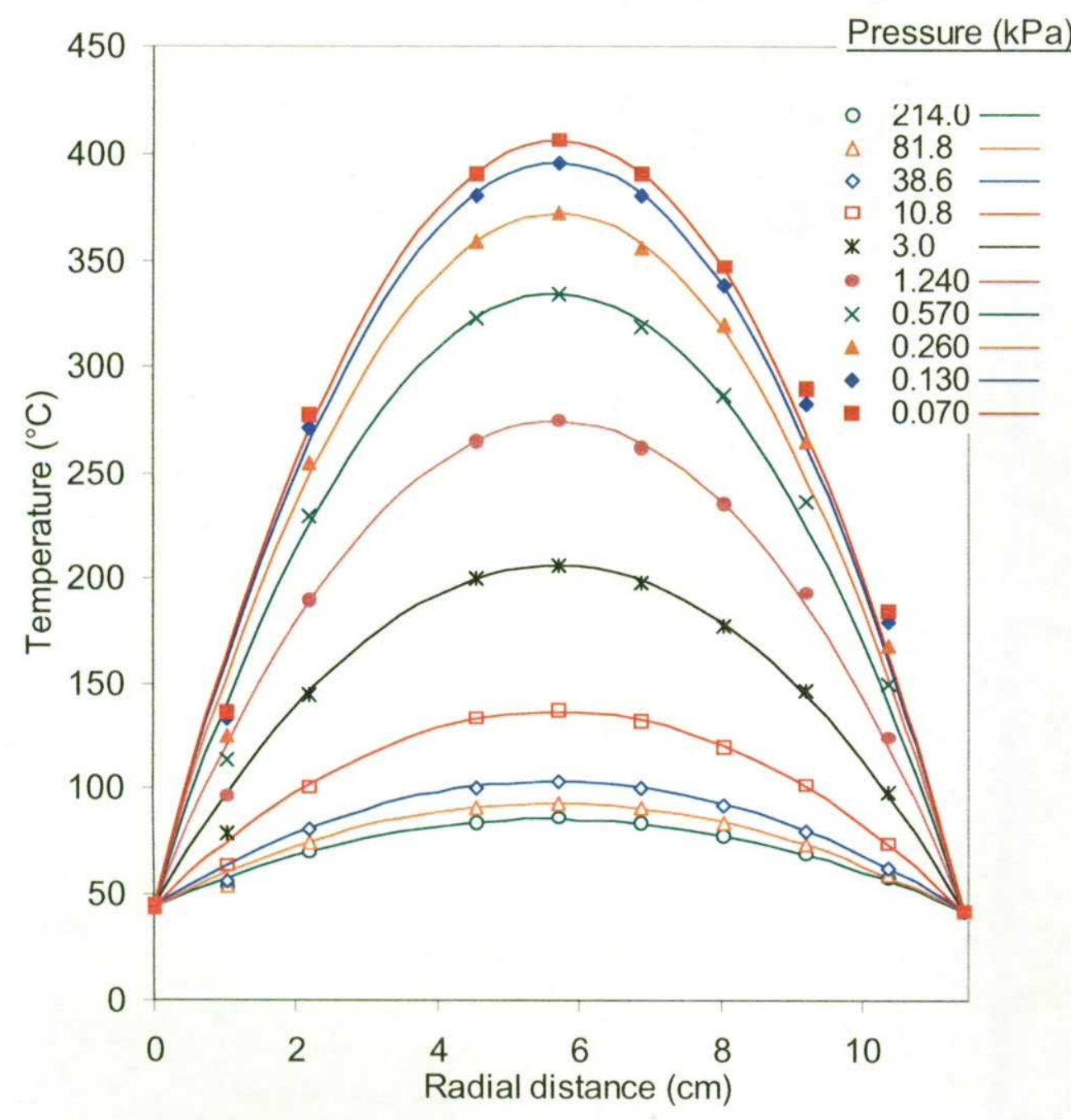

Calculated effective thermal conductivities

$$
\begin{aligned}
& \begin{array}{|r|r|r|}
\hline \begin{array}{c}
\boldsymbol{P} \\
(\mathbf{k P a})
\end{array} & \begin{array}{c}
\Delta \boldsymbol{T}_{\text {bed }} \\
\left({ }^{\circ} \mathbf{C}\right)
\end{array} & \begin{array}{c}
\boldsymbol{k}_{\text {eff }} \\
(\mathbf{W} / \mathbf{m}-\mathbf{K})
\end{array} \\
\hline 214.0 & 44 & 0.090 \\
\hline 81.8 & 52 & 0.077 \\
\hline 38.6 & 61 & 0.064 \\
\hline 10.8 & 95 & 0.041 \\
\hline 3.0 & 164 & 0.024 \\
\hline 1.240 & 233 & 0.017 \\
\hline 0.570 & 292 & 0.013 \\
\hline 0.260 & 330 & 0.012 \\
\hline 0.130 & 354 & 0.011 \\
\hline 0.070 & 364 & 0.011 \\
\hline
\end{array} \\
& T(r)=-\frac{\dot{q} r^{2}}{4}+T(r=0) \\
& k_{\text {eff }} \Delta T_{\text {bed }}=\frac{\dot{q} R^{2}}{4}
\end{aligned}
$$




\section{Pressure dependence of $k_{\text {eff }}$}

- Gas thermal conductivity $\left(k_{g}\right)$ is pressure dependent depending on operating regime

- Operating regime determined by Knudsen number $(\mathrm{Kn})$

$$
\begin{aligned}
& \mathrm{Kn}=l / \lambda \\
&-\quad \lambda=\text { pore size } \\
&-\quad l=\text { mean free path } \\
& l=\frac{k_{B} T}{\sqrt{2} \pi D_{m}^{2} P}
\end{aligned}
$$

- Gas conductivities:

$$
\begin{aligned}
& k_{\text {cont }}=f(T) \text { only } \\
& k_{f m}=\left(\frac{\alpha}{2-\alpha}\right) \lambda \Lambda_{o} P \sqrt{\frac{T_{r e f}}{T}}
\end{aligned}
$$

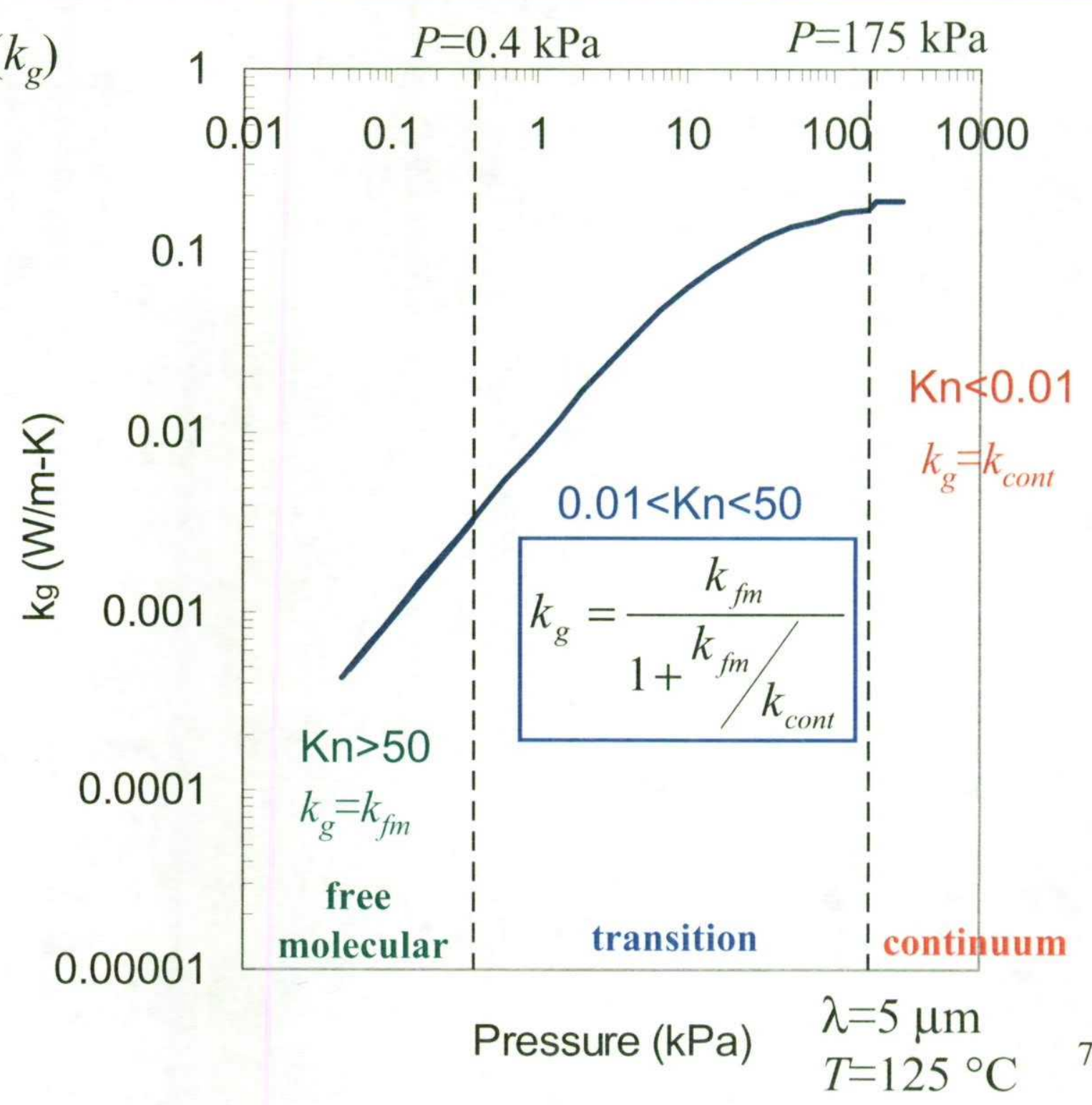




\section{$\mathrm{He}$ and Ar centerline temperatures}

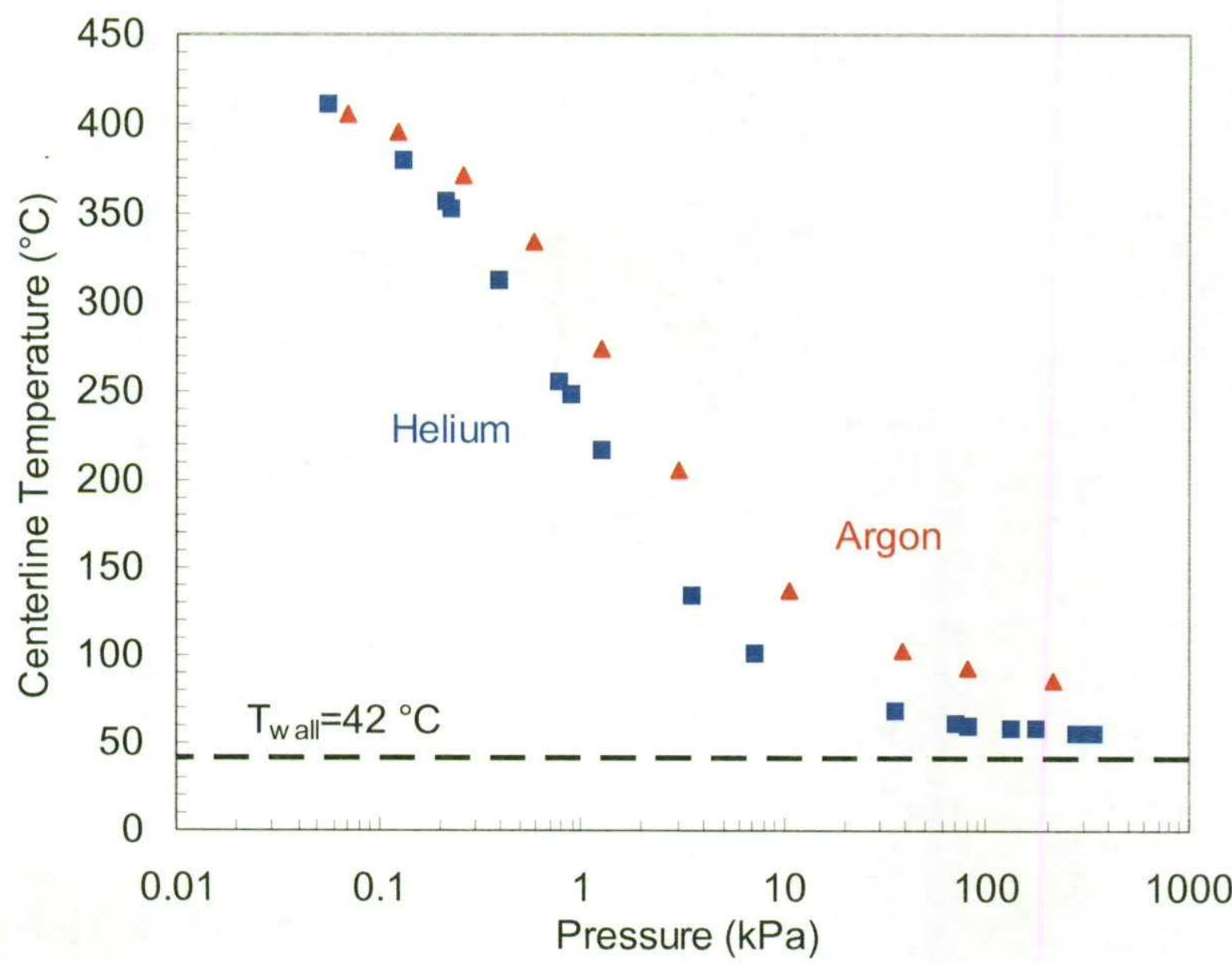

\begin{tabular}{|c|c|c|c|}
\hline \multicolumn{2}{|c|}{ Helium } & \multicolumn{2}{|c|}{ Argon } \\
\hline$P(\mathrm{kPa})$ & $\begin{array}{c}k_{\text {eff }} \\
(\mathrm{W} / \mathrm{m}-\mathrm{K})\end{array}$ & $P(\mathrm{kPa})$ & $\begin{array}{c}k_{\text {eff }} \\
(\mathrm{W} / \mathrm{m}-\mathrm{K})\end{array}$ \\
\hline 334.4 & 0.29 & 214.0 & 0.090 \\
\hline 277.6 & 0.28 & 81.8 & 0.077 \\
\hline 173.2 & 0.24 & 38.6 & 0.064 \\
\hline 130.4 & 0.24 & 10.8 & 0.041 \\
\hline 82.2 & 0.22 & 3.0 & 0.024 \\
\hline 69.8 & 0.20 & 1.240 & 0.017 \\
\hline 35.0 & 0.15 & 0.570 & 0.013 \\
\hline 7.0 & 0.066 & 0.260 & 0.012 \\
\hline 3.6 & 0.043 & 0.130 & 0.011 \\
\hline 1.2 & 0.022 & 0.070 & 0.011 \\
\hline 0.890 & 0.019 & & \\
\hline 0.770 & 0.018 & & \\
\hline 0.390 & 0.015 & & \\
\hline 0.225 & 0.013 & & \\
\hline 0.210 & 0.012 & & \\
\hline 0.130 & 0.012 & & \\
\hline 0.055 & 0.011 & & \\
\hline
\end{tabular}

$\boldsymbol{k}_{\text {eff }}$ predictions from D-E eqn.:

$$
\begin{aligned}
& k_{e f f, H e}=0.31 \mathrm{~W} / \mathrm{mK} \\
& k_{e f f, A r}=0.063 \mathrm{~W} / \mathrm{mK}
\end{aligned}
$$




\section{New model development}

- Effective thermal conductivity models in the literature do not correctly predict our experimental results

- the $\mathrm{PuO}_{2}$ powder contains random shaped particles with a high porosity

- many models make limiting assumptions, such as equal sized spheres with a packing fraction of 0.475

- most models predict that $k_{e f f, H e}=5^{*} k_{\text {eff, }, A r}$ at high pressure. From our experimental measurements, $k_{\text {eff, } \mathrm{He}}=3^{*} k_{\text {eff, } A r}$

- Developed a model with heat conduction through gas and solid pathways in parallel and in series

- primary difference from similar models in literature ${ }^{4,5}$ : no limiting assumptions about powder porosity or particle shape 


\section{Thermal conductivity model}

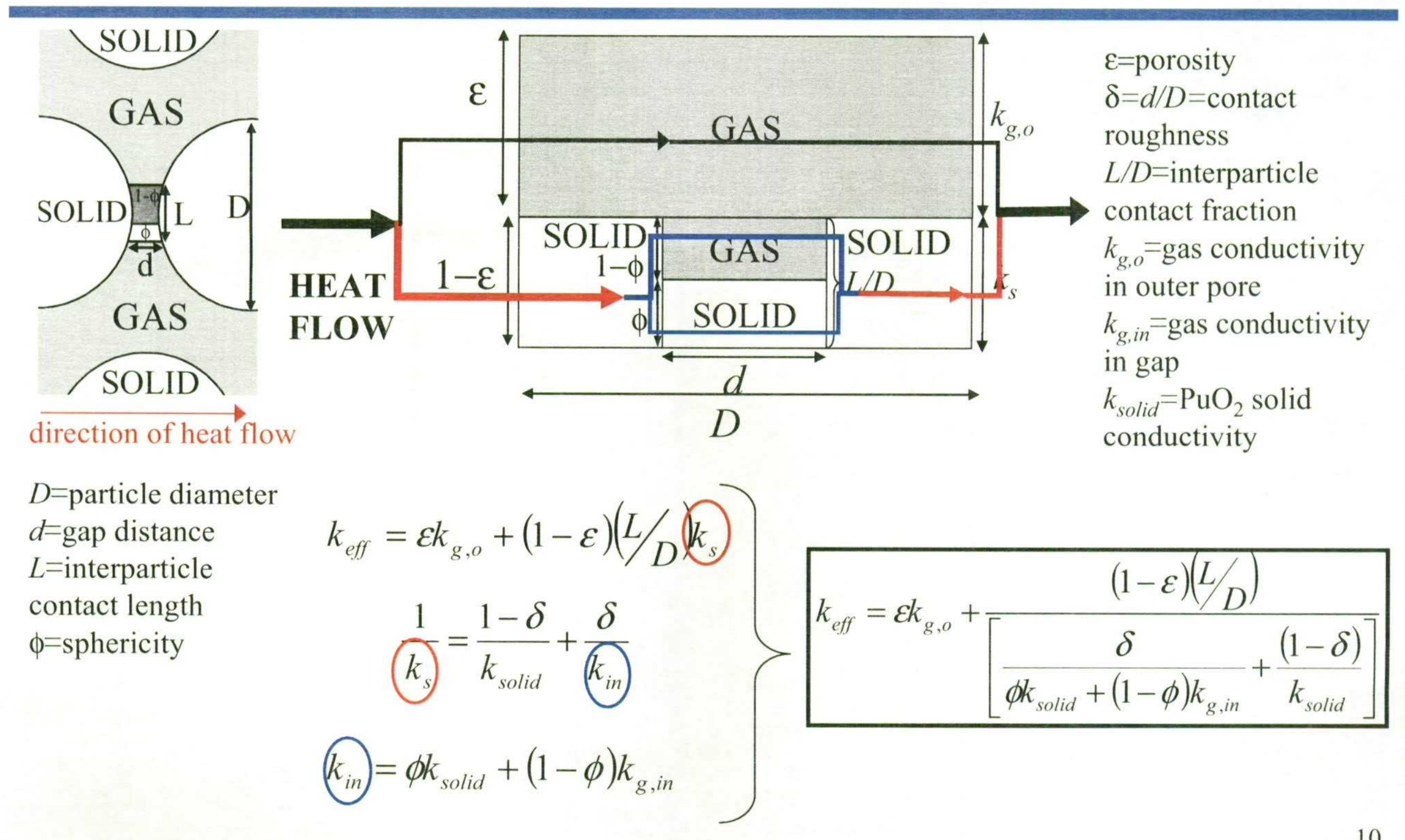




\section{Parameters to calculate $k_{\text {eff }}$}

- To calculate $k_{\text {eff }}$ we need to estimate:

- the parameters $\delta, \phi$, and $L / D$

- the pore sizes $\left(\lambda_{o}\right.$ and $\left.\lambda_{i n}\right)$ to calculate $k_{g, o}$ and $k_{g, i n}$ for each

\begin{tabular}{ll} 
Parameter & Value \\
\hline$\delta$ & $3.7 \times 10^{-3}$ \\
$\phi$ & $1.8 \times 10^{-4}$ \\
$L / D$ & 0.10 \\
$\lambda_{\text {in }}(\mu \mathrm{m})$ & 0.68 \\
$\lambda_{o}(\mu \mathrm{m})$ & 17 \\
\hline
\end{tabular}
gas

$$
k_{\text {eff }}=\varepsilon k_{g, o}+\frac{(1-\varepsilon)(L / D)}{\left[\frac{\underline{\delta}}{\phi k_{\text {solid }}+(1-\underline{\phi}) \underline{k_{g, i n}}}+\frac{(1-\underline{\delta})}{k_{\text {solid }}}\right]}
$$

$k_{g}$ free molecular expressions

$$
\begin{aligned}
& k_{g A r, o}=\left(\frac{\alpha_{A r}}{2-\alpha_{A r}}\right) \lambda_{o} \Lambda_{A r} P \sqrt{\frac{T_{r e f}}{T}} \\
& k_{g A r, i n}=\left(\frac{\alpha_{A r}}{2-\alpha_{A r}}\right) \lambda_{\underline{i n}} \Lambda_{A r} P \sqrt{\frac{T_{r e f}}{T}}
\end{aligned}
$$

\begin{tabular}{|l|l|l|}
\hline $\begin{array}{l}\text { Gas-dependent } \\
\text { parameters }\end{array}$ & $\mathrm{Ar}$ & $\mathrm{He}$ \\
\hline$\alpha$ & 0.9 & 0.5 \\
\hline$\Lambda\left(\mathrm{W} / \mathrm{m}^{2}-\mathrm{K}-\mathrm{Pa}\right)$ & 0.697 & 2.202 \\
\hline
\end{tabular}




\section{Modeling results: importance of interparticle contact area}

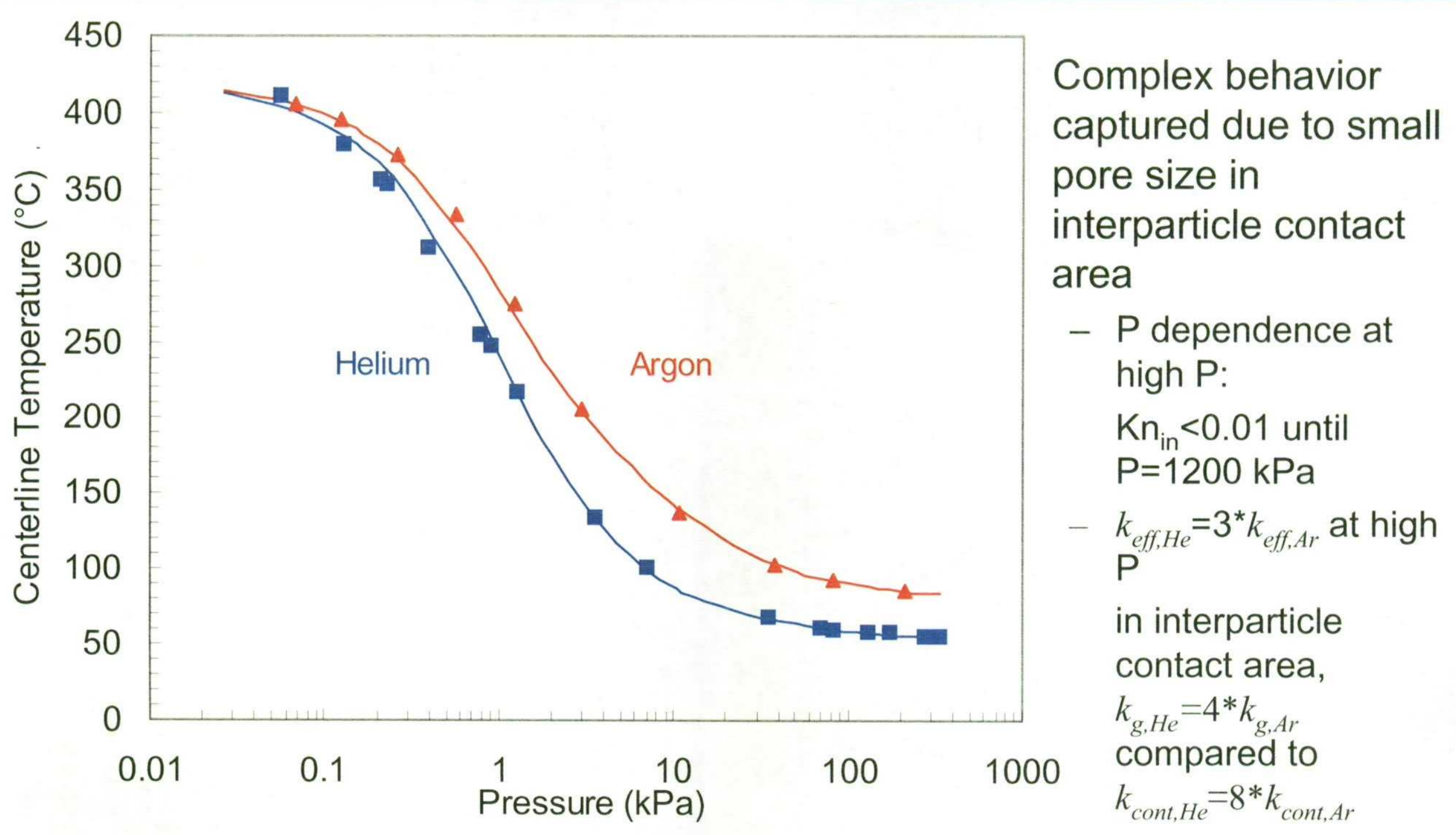




\section{Radial profile results for argon}

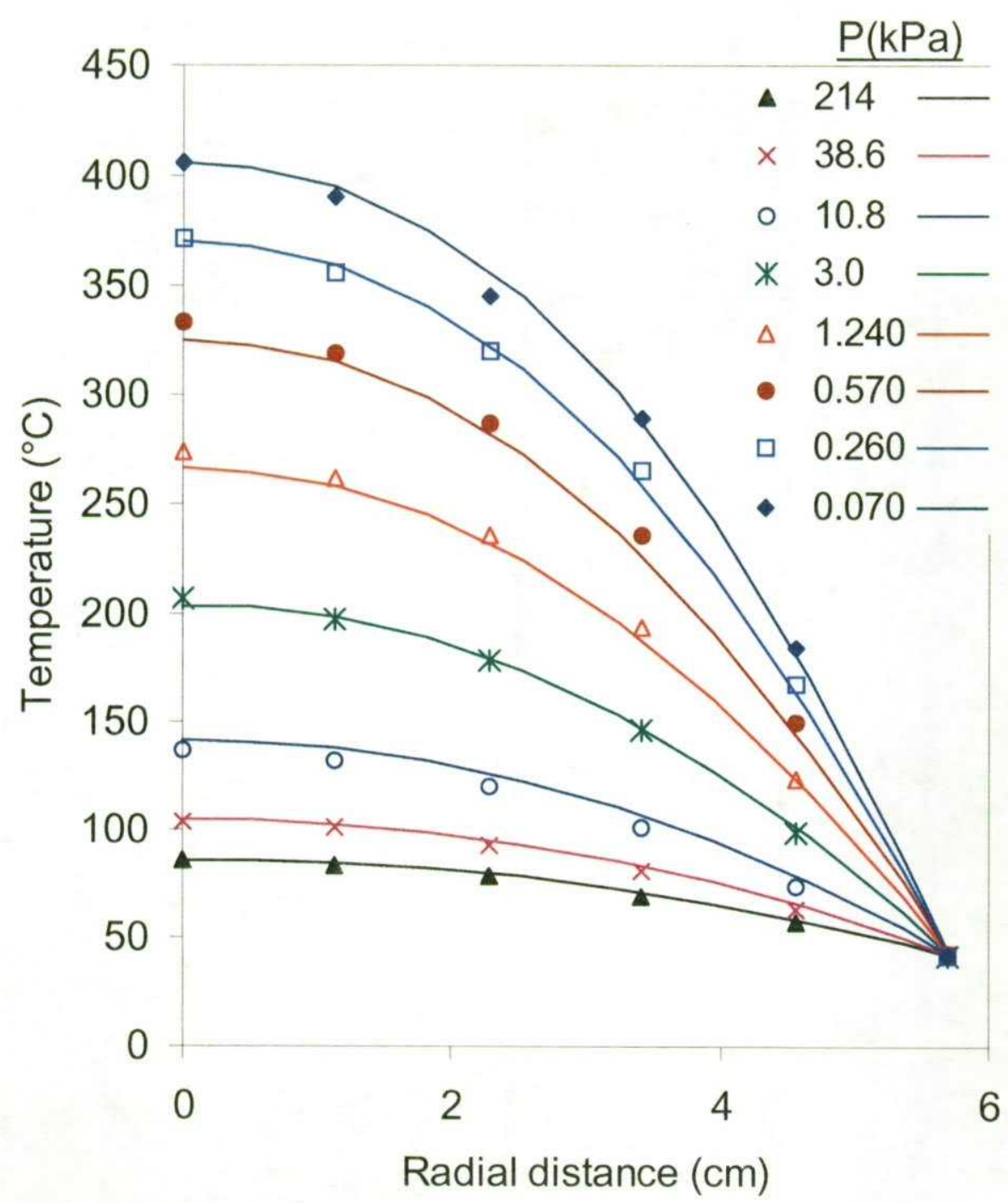

- Predicted radial profiles agree well with expt. measurements at all pressures

- Indicates that contributions of thermal radiation and heat conduction are correctly captured by model

- thermal radiation most important at high $\mathrm{T}$ ( $\mathrm{T}^{4}$ dependence)

- at low $P$, thermal radiation important in center of container and solid-solid conduction dominates near container wall 


\section{Conclusions}

- Measured temperature profiles of $\mathrm{PuO}_{2}$ powders at varying pressures with argon and helium fill gases

- Developed thermal conductivity model that agrees well with experimental data

- determined that including interparticle area and pore size was necessary to predict high pressure powder behavior

- thermal conductivity expression can be used for a wide variety of powders

- Compared predictions from new conductivity expression with thermal conductivity values used in previous $\mathrm{PuO}_{2}$ modeling studies

- previous $\mathrm{PuO}_{2}$ thermal conductivity value:

- $k_{\text {eff }}=0.0796 \mathrm{~W} / \mathrm{m}-\mathrm{K}$ for air

- predicted value with new model:

- $k_{\text {eff }}=0.155 \mathrm{~W} / \mathrm{m}-\mathrm{K}$ for air

- $\Delta \mathrm{T}$ across $\mathrm{PuO}_{2}$ bed decreases by approximately half 


\section{Acknowlegements}

- Experimental measurements:

- John Berg, Dennis Padilla, Alex Carrillo, Laura Worl: LANL, Nuclear Materials Technology Division

- David Harradine: LANL, Chemistry Division

- Thad Knight: LANL, Decision Applications Division

- Funding:

- Nuclear Materials Stabilization Program Office, United States Department of Energy, Albuquerque Operations and Headquarters Offices under the auspices of the DNFSB 94-1 Research and Development Project 